\title{
Relative Lengths of Paths and Cycles in 2-Connected Graphs
}

\author{
Zhora G. Nikoghosyan \\ Institute for Informatics and Automation Problems of NAS RA \\ e-mail: zhora@ipia.sci.am
}

\begin{abstract}
Let $l$ be the length of a longest path in a 2-connected graph $G$ and $c$ the circumference - the length of a longest cycle in $G$. In 1952, Dirac proved that $c>\sqrt{2 l}$, by noting that "actually $c \geq 2 \sqrt{l}$, but the proof of this result, which is best possible, is rather complicated". Let $L_{1}, L_{2}, \ldots, L_{m}$ be a vine on a longest path of $G$. In this paper, using the parameter $\mathrm{m}$, we present a more general sharp bound for the circumference $c$ including the bound $c \geq 2 \sqrt{l}$ as an immediate corollary, based on elementary arguments.
\end{abstract}

Keywords: Longest cycle, Longest path, Circumference, Vine.

\section{Introduction}

We consider only undirected graphs with no loops or multiple edges. Let $G$ be a 2-connected graph. We use $c$ and $l$ to denote the circumference (the length of a longest cycle) and the length (the number of edges) of a longest path of $G$. A good reference for any undefined terms is [1].

In 1952, Dirac [2] proved the following.

Theorem A: [2]. In every 2-connected graph, $c>\sqrt{2 l}$.

In the same paper [2], Dirac considered a sharp version of Theorem A by noting that "actually $c \geq 2 \sqrt{l}$, but the proof of this result, which is best possible, is rather complicated". Analogous questions were studied for $k$-connected graphs when $k \geq 3$ by Bondy and Locke $([4],[5])$.

In this paper, using a new parameter, we present a more general sharp bound for the circumference $\mathrm{c}$ in 2-connected graphs in terms of $l$ and the length of a vine on a longest path of $G$, including the bound $c \geq 2 \sqrt{l}$ as a corollary, based on elementary arguments. In order to formulate this result, we need some additional definitions and notations.

The set of vertices of a graph $G$ is denoted by $V(G)$ and the set of edges by $E(G)$. If $Q$ is a path or a cycle, then the length of $Q$, denoted by $l(Q)$, is $|E(Q)|$ - the number of edges in $Q$. We write a cycle $Q$ with a given orientation by $\vec{Q}$. For $x, y \in V(Q)$, we denote by 
$x \vec{Q} y$ the subpath of $Q$ in the chosen direction from $x$ to $y$. We use $P=x \vec{P} y$ to denote a path with end vertices $x$ and $y$ in the direction from $x$ to $y$. We say that vertex $z_{1}$ precedes vertex $z_{2}$ on $\vec{Q}$ if $z_{1}, z_{2}$ occur on $\vec{Q}$ in this order, and indicate this relationship by $z_{1} \prec z_{2}$. We will write $z_{1} \preceq z_{2}$ when either $z_{1}=z_{2}$ or $z_{1} \prec z_{2}$.

Let $P=x \vec{P} y$ be a path. A vine of length $m$ on $P$ is a set

$$
\left\{L_{i}=x_{i} \vec{L}_{i} y_{i}: 1 \leq i \leq m\right\}
$$

of internally-disjoint paths such that

(a) $V\left(L_{i}\right) \cap V(P)=\left\{x_{i}, y_{i}\right\} \quad(i=1, \ldots, m)$,

(b) $x=x_{1} \prec x_{2} \prec y_{1} \preceq x_{3} \prec y_{2} \preceq x_{4} \prec \ldots \preceq x_{m} \prec y_{m-1} \prec y_{m}=y$ on $P$.

The main result is the following.

Theorem 1: Let $G$ be a 2-connected graph. If $\left\{L_{1}, L_{2}, \ldots, L_{m}\right\}$ is a vine on a longest path of $G$, then

$$
c \geq\left\{\begin{array}{l}
\frac{2 l}{m+1}+\frac{m+1}{2}, \text { when } m \text { is odd, } \\
\frac{2 l-\frac{1}{2}}{m+1}+\frac{m+1}{2}, \text { when } m \text { is even. }
\end{array}\right.
$$

Equivalently, Theorem 1 can be formulated as follows, implying Dirac's conjecture as an immediate corollary.

Theorem 2: Let $G$ be a 2-connected graph. If $\left\{L_{1}, L_{2}, \ldots, L_{m}\right\}$ is a vine on a longest path of $G$, then

$$
c \geq \begin{cases}\sqrt{4 l+(c-m-1)^{2}}, & \text { when } m \text { is odd, } \\ \sqrt{4 l+(c-m-1)^{2}-1}, & \text { when } m \text { is even. }\end{cases}
$$

Corollary 1: In every 2-connected graph, $c \geq 2 \sqrt{l}$.

Note that if $m$ is odd, then $c>2 \sqrt{l}$.

The following lemma guarantees the existence of at least one vine on a longest path in a 2-connected graph.

The Vine Lemma: [3]. Let $G$ be a k-connected graph and $P$ a path in $G$. Then there are $k-1$ pairwise-disjoint vines on $P$.

\section{Proofs}

Proof of Theorem 1. Let $P=x \vec{P} y$ be a longest path in $G$ and let

$$
\left\{L_{i}=x_{i} \vec{L}_{i} y_{i}: 1 \leq i \leq m\right\}
$$

be a vine of length $m$ on $P$. Put 


$$
\begin{gathered}
L_{i}=x_{i} \vec{L}_{i} y_{i} \quad(i=1, \ldots, m), \quad A_{1}=x_{1} \vec{P} x_{2}, \quad A_{m}=y_{m-1} \vec{P} y_{m}, \\
A_{i}=y_{i-1} \vec{P} x_{i+1} \quad(i=2,3, \ldots, m-1), \\
B_{i}=x_{i+1} \vec{P} y_{i} \quad(i=1, \ldots, m-1), \\
l\left(A_{i}\right)=a_{i} \quad(i=1, \ldots, m), \quad l\left(B_{i}\right)=b_{i} \quad(i=1, \ldots, m-1) .
\end{gathered}
$$

Using the given vine $L_{1}, L_{2}, \ldots, L_{m}$, we construct a number of appropriate cycles and obtain a lower bound for the circumference as a mean of their lengths. First, we put

$$
\begin{gathered}
Q_{0}=\bigcup_{i=1}^{m}\left(A_{i} \cup L_{i}\right), \\
Q_{i}=\bigcup_{j=i+1}^{m-i}\left(A_{j} \cup L_{j}\right) \cup B_{i} \cup B_{m-i},
\end{gathered}
$$

where $i \in\{1,2, \ldots,(m-1) / 2\}$ when $m$ is odd, and $i \in\{1,2, \ldots,(m-2) / 2\}$ when $m$ is even. Since $l\left(L_{i}\right) \geq 1 \quad(i=1,2, \ldots, m)$ and $a_{1} \geq 1, a_{m} \geq 1$, we have

$$
c \geq l\left(Q_{0}\right)=\sum_{i=1}^{m} l\left(L_{i}\right)+a_{1}+a_{m}+\sum_{i=2}^{m-1} a_{i} \geq m+a_{1}+a_{m} .
$$

Case 1. $m$ is odd.

For each $i \in\{1,2, \ldots,(m-1) / 2\}$, we have

$$
\begin{gathered}
c \geq l\left(Q_{i}\right)=b_{i}+b_{m-i}+\sum_{j=i+1}^{m-i}\left(a_{j}+l\left(L_{j}\right)\right) \\
\geq b_{i}+b_{m-i}+\sum_{j=i+1}^{m-i} a_{j}+m-2 i .
\end{gathered}
$$

By summing (1) and (2), we get

$$
\frac{m+1}{2} c \geq \sum_{i=0}^{\frac{m-1}{2}} l\left(Q_{i}\right) \geq \sum_{i=1}^{m-1} b_{i}+\sum_{i=1}^{m} a_{i}+\frac{m+1}{2} m-2 \sum_{i=1}^{\frac{m-1}{2}} i .
$$

Since $l=\sum_{i=1}^{m-1} b_{i}+\sum_{i=1}^{m} a_{i}$, we have

$$
\frac{m+1}{2} c \geq l+\frac{m+1}{2} m-\frac{m^{2}-1}{4}=l+\frac{(m+1)^{2}}{4}
$$

implying that

$$
c \geq \frac{2 l}{m+1}+\frac{m+1}{2}
$$

Case 2. $m$ is even.

As in Case 1 , for each $i \in\{1,2, \ldots,(m-2) / 2\}$,

$$
c \geq l\left(Q_{i}\right) \geq b_{i}+b_{m-i}+\sum_{j=i+1}^{m-i} a_{j}+m-2 i .
$$


Case 2.1. $\frac{1}{2} c \geq b \frac{m}{2}$.

By summing (1), (3) and $\frac{1}{2} c \geq b \frac{m}{2}$, we get

$$
\begin{gathered}
\frac{m}{2} c+\frac{1}{2} c \geq\left(\sum_{i=1}^{m-1} b_{i}+\sum_{i=1}^{m} a_{i}\right)+\sum_{i=0}^{\frac{m-2}{2}}(m-2 i) \\
=l+\frac{m}{2} m-2 \sum_{i=0}^{\frac{m-2}{2}} i=l+\frac{m(m+2)}{4},
\end{gathered}
$$

implying that

$$
c \geq \frac{2 l-\frac{1}{2}}{m+1}+\frac{m+1}{2} .
$$

Case 2.2. $\frac{1}{2}(c+1) \leq b \frac{m}{2}$.

Put

$$
\begin{gathered}
R_{0}=B_{\frac{m}{2}} \cup \bigcup_{i=1}^{\frac{m}{2}}\left(A_{i} \cup L_{i}\right), \\
R_{m}=B_{\frac{m}{2}} \cup \bigcup_{i=\frac{m+2}{2}}^{m}\left(A_{i} \cup L_{i}\right) .
\end{gathered}
$$

Further, for each $i \in\left\{1,2, \ldots, \frac{m-2}{2}\right\}$, we put

$$
\begin{gathered}
R_{i}=B_{\frac{m}{2}} \cup B_{i} \cup \bigcup_{j=i+1}^{\frac{m}{2}}\left(A_{j} \cup L_{j}\right), \\
R_{m-i}=B_{\frac{m}{2}} \cup B_{m-i} \cup \bigcup_{j=\frac{m+2}{2}}^{m-i}\left(A_{j} \cup L_{j}\right) .
\end{gathered}
$$

Then clearly,

$$
\begin{gathered}
c \geq l\left(R_{0}\right)=b \frac{m}{2}+\sum_{i=1}^{\frac{m}{2}}\left(a_{i} \cup l\left(L_{i}\right)\right) \geq b_{\frac{m}{2}}+\sum_{i=1}^{\frac{m}{2}} a_{i}+\frac{m}{2}, \\
c \geq l\left(R_{m}\right)=b \frac{m}{2}+\sum_{i=\frac{m+2}{2}}^{m}\left(a_{i} \cup l\left(L_{i}\right)\right) \\
\geq b \frac{m}{2}+\sum_{i=\frac{m+2}{2}}^{m} a_{i}+\frac{m}{2} .
\end{gathered}
$$

Furthermore, for each $i \in\left\{1,2, \ldots, \frac{m-2}{2}\right\}$,

$$
\begin{aligned}
c \geq l\left(R_{i}\right) & =b_{\frac{m}{2}}+b_{i}+\sum_{j=i+1}^{\frac{m}{2}}\left(a_{j}+l\left(L_{j}\right)\right) \\
& \geq b_{\frac{m}{2}}+b_{i}+\frac{m}{2}-i
\end{aligned}
$$




$$
\begin{aligned}
c \geq l\left(R_{m-i}\right) & =b_{\frac{m}{2}}+b_{m-i}+\sum_{j=\frac{m+2}{2}}^{m-i}\left(a_{j}+l\left(L_{j}\right)\right) \\
& \geq b_{\frac{m}{2}}+b_{m-i}+\frac{m}{2}-i .
\end{aligned}
$$

By summing (4), (5), (6) and (7), we get

$$
\begin{gathered}
m c \geq m b_{\frac{m}{2}}+\sum_{i=1}^{m} a_{i}+\left(\sum_{i=1}^{m-1} b_{i}-b_{\frac{m}{2}}\right)+m \frac{m}{2}-2 \sum_{i=1}^{\frac{m-2}{2}} i \\
=(m-1) b_{\frac{m}{2}}+\left(\sum_{i=1}^{m} a_{i}+\sum_{i=1}^{m-1} b_{i}\right)+\frac{m^{2}+2 m}{4} \\
\geq \frac{(m-1)(c+1)}{2}+l+\frac{m^{2}+2 m}{4}
\end{gathered}
$$

implying that

$$
c \geq \frac{2 l}{m+1}+\frac{(m+2)^{2}-6}{2(m+1)} \geq \frac{2 l-\frac{1}{2}}{m+1}+\frac{m+1}{2} .
$$

Theorem 1 is proved.

Proof of Theorem 2. By (1), $c \geq m+a_{1}+a_{2} \geq m+2$. Let $c=m+y+2$ for some integer $y \geq 0$. By substituting $m=c-y-2$ in Theorem 1 , we get

$$
c \geq \sqrt{4 l+(y+1)^{2}}=\sqrt{4 l+(c-m-1)^{2}}
$$

when $m$ is odd; and

$$
c \geq \sqrt{4 l+y^{2}}=\sqrt{4 l+(c-m-2)^{2}}
$$

when $m$ is even. Theorem 2 is proved.

To show the sharpness of the bounds in Theorems 1 and 2, let $P=x \vec{P} y$ be a path and let

$$
\left\{L_{i}=x_{i} \vec{L}_{i} y_{i}: 1 \leq i \leq m\right\}
$$

be a vine on $P$. Put

$$
\begin{gathered}
L_{i}=x_{i} \vec{L}_{i} y_{i} \quad(i=1, \ldots, m), \quad A_{1}=x_{1} \vec{P} x_{2}, \quad A_{m}=y_{m-1} \vec{P} y_{m}, \\
A_{i}=y_{i-1} \vec{P} x_{i+1} \quad(i=2,3, \ldots, m-1), \quad B_{i}=x_{i+1} \vec{P} y_{i} \quad(i=1, \ldots, m-1), \\
l\left(A_{i}\right)=a_{i}(i=1, \ldots, m), \quad l\left(B_{i}\right)=b_{i} \quad(i=1, \ldots, m-1) .
\end{gathered}
$$

Let $y \geq 0$ by an integer and

$$
\begin{gathered}
a_{1}=a_{m}=\frac{y}{2}+1, a_{2}=a_{3}=\ldots=a_{m-1}=0, \\
b_{i}=b_{m-i}=\frac{y}{2}+i+1(i=1,2, \ldots,\lfloor(m-1) / 2\rfloor) .
\end{gathered}
$$


If $m$ is odd, then it is easy to see that

$$
c=m+y+2=\frac{2 l}{m+1}+\frac{m+1}{2}=\sqrt{4 l+(c-m-1)^{2}} .
$$

If $m$ is even, we put $b_{m / 2}=\frac{y}{2}+\frac{m+2}{2}$, implying that

$$
c=m+y+2=\frac{2 l-\frac{1}{2}}{m+1}+\frac{m+1}{2}=\sqrt{4 l+(c-m-1)^{2}-1} .
$$

Thus, the bounds in Theorems 1 and 2 are best possible.

\title{
References
}

[1] J.A. Bondy and U.S.R. Murty, Graph Theory with Applications, Macmillan, London and Elsevier, New York, 1976.

[2] G.A. Dirac, "Some theorems on abstract graphs", Proc. London, Math. Soc., vol. 2, pp. 69-81, 1952.

[3] J. A. Bondy, Basic Graph Theory: Paths and Circuits, Handbook of combinatorics, vol. 1,2, Elsevier, Amsterdam, 1990.

[4] J. A. Bondy and S. C. Locke, "Relative lengths of paths and cycles in $k$-connected graphs", Annals of Discrete Mathematics, vol. 8, pp. 253-259, 1980.

[5] S.C. Locke, "Relative lengths of paths and cycles in k-connected graphs", J. of Comb. Theory, Series B 32, pp. 206-222, 1982.

Submitted 25.05.2019, accepted 10.10.2019.

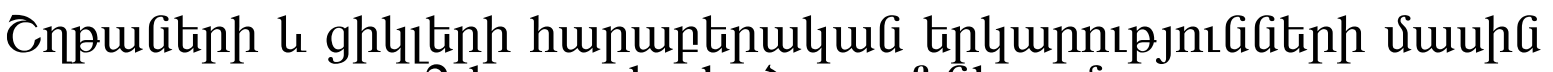 2-luuquigumd qpuфquipniर}

\author{
đnpu \%. ઉplnnnujua

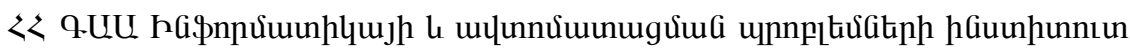 \\ e-mail: zhora@ipia.sci.am
}

\section{Uựnnนnıu}

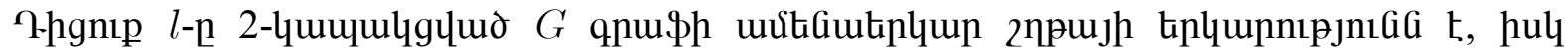

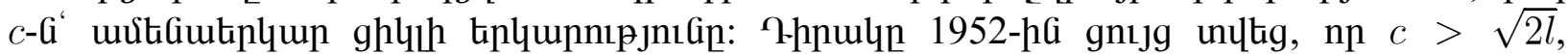

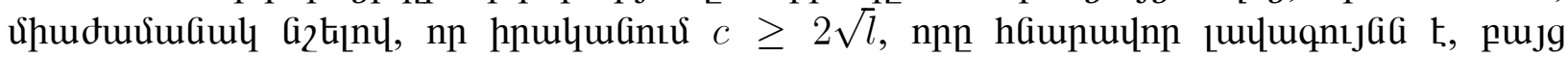

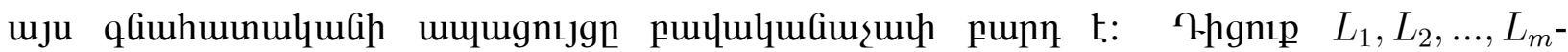

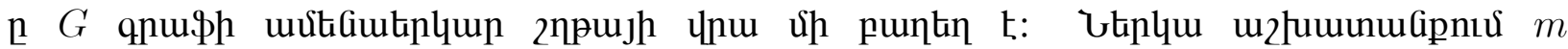

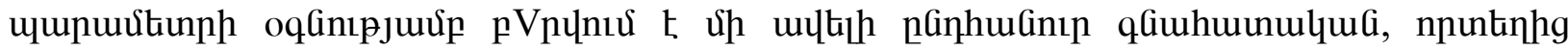




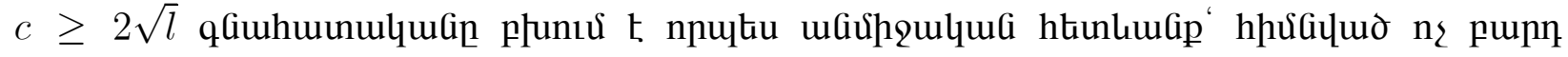
nuunnnıpjnıGiliph цnu:

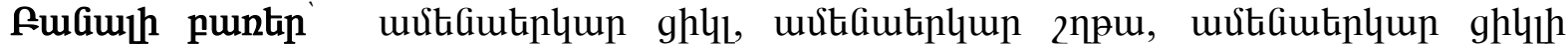
tiplupnıpjnıG, puntin:

\title{
Относительные Алины цепей и циклов в 2-связных графах
}

\author{
Жора Г. Никогосян \\ Институт проблем информатики и автоматизации НАН РА \\ e-mail: zhora@ipia.sci.am
}

\begin{abstract}
Аннотация
Пусть $l$ обозначает длину Алиннейшей цепи графа $G$, а с обозначает длину Алиннейшего цикла. В 1952г. Аирак доказал, что $c>\sqrt{2 l}$, отметив, что "в самом деле имеет место $c \geq 2 \sqrt{l}$, что улучшить невозможно, но доказательство этой оценки достаточно сложно". Пусть $L_{1}, L_{2}, \ldots, L_{m}$ - плющ на длиннейшей цепи графа $G$. В настоящей работе приводится новая более общая оценка, откуда вытекает справедливость оценки $c \geq 2 \sqrt{l}$ как непосредственное следствие, основанное на элэментарных соображений.
\end{abstract}

Ключевые слова: длиннейший цикл, Алинннейшая цепь, длина длиннейшего цикла, плющ. 